Preprint typeset in JHEP style - HYPER VERSION

\title{
Dark Matter Annihilation and the PAMELA, FERMI and ATIC Anomalies
}

\author{
A. A. El-Zant ${ }^{1}$, S. Khalil ${ }^{1,2}$, and H. Okada ${ }^{1}$ \\ ${ }^{1}$ Centre for Theoretical Physics, The British University in Egypt, El Sherouk City, \\ Postal No, 11837, P.O. Box 43, Egypt \\ ${ }^{2}$ Department of Mathematics, Ain Shams University, Faculty of Science, Cairo, 11566, \\ Egypt
}

ABSTRACT: If dark matter (DM) annihilation accounts for the tantalizing excess of cosmic ray electron/positrons, as reported by the PAMELA, ATIC, HESS and FERMI observatories, then the implied annihilation cross section must be relatively large. This results, in the context of standard cosmological models, in very small relic DM abundances that are incompatible with astrophysical observations. We explore possible resolutions to this apparent conflict in terms of non-standard cosmological scenarios; plausibly allowing for large cross sections, while maintaining relic abundances in accord with current observations.

KEYWORDs: Low scale $B-L$, Dark Matter. 


\section{Contents}

1. Introduction 1

2. PAMELA, ATIC, FERMI and HESS Anomalies 2

3. Relic Abundance and Non-conventional Cosmology 5

4. Extra Constraints on the Annihilation Cross Section and the Viability of our Model 9

5. Conclusions 10

\section{Introduction}

The context of our investigation is set by the overwhelming evidence for a non-baryonic component dominating the matter content of our Universe, as inferred from a combination of colluding astrophysical phenomena (the dynamics of galaxies and clusters; large scale structure in the Universe; cosmic microwave background fluctuations; big bang nucleosynthesis). Though relying exclusively on large scale gravitational signatures, the implied existence of DM alludes to microscopic fundamental physics beyond the standard model. But although there are several ongoing attempts at direct detection of expected DM candidates, apart from the controversial results of DAMA collaboration [1] and recent tentative data points emanating from the CDMSII experiment [2], their outcomes have hitherto been invariably negative. It is in this context that the significant excitement concerning the recent measurements of excess electron and positron flux, as reported by the PAMELA, ATIC, HESS and FERMI collaborations, among others, arose.

PAMELA's observations, reporting excess flux between 8 and $80 \mathrm{GeV}$ [3], with no excess in the corresponding anti-proton flux, confirm and extend previous results obtained by HEAT [4] and AIMS [5]. The ATIC [6] experiment data, on the other hand, show significant excess electron and positron flux at energies around $300-800 \mathrm{GeV}$.

Although the PAMELA data is marginally consistent with calculations of the cosmic ray background employing a 'soft' background electron flux spectrum [7], the ATIC and FERMI observations more clearly point to the existence of an additional source of high energy electrons and positrons; and since such particles cannot travel very far without much energy loss, their source must be local ( $\lesssim 1 \mathrm{kpc}$ from the solar system). There are plausible astrophysical explanations for this excess too, e.g. in terms of local pulsars and supernovas remnants [8] [9], but they seem less natural than when invoked in the low energy region of the spectrum. The excesses in flux could also result from DM annihilation 
or decay, and a vast literature has recently arisen around the subject (see, e.g., [10] [11] for a review), partly instigated by a salient feature (perhaps especially prominent in the ATIC measurements) of the cosmic ray positron spectrum produced by DM annihilation; the fact that it drops off at $E_{e^{+}}=m_{\chi}$, while the flux produced by, e.g., a single pulsars falls off more gradually. Though this feature seems significantly more subdued in the FERMI data [12], the relevant region is relatively less well represented there - at least in comparison with the HESS data, where a palpable drop-off is present [13], [14]. Nevertheless, is difficult to conclusively differentiate between astrophysical and DM descriptions of the observed positron excesses in any conclusive manner, although future experiments may be able to achieve this. One advantage of DM models is that, despite their diversity, their cosmic rays emanate from far simpler physical objects and are therefore far easier to falsify than those pertaining to astrophysical sources.

In any case, if the DM scenario is to explain the observed anomalous flux, one major hurdle has to be surpassed. At present the major difficulty facing the DM annihilation is the large cross section apparently required to fit the excess flux - one that seems incompatible with straightforward estimates of the relic DM abundance in conventional cosmological models.

This paper is an attempt at reconciling the aforementioned observed cosmic ray excess with an interpretation of the results in terms of annihilating halo dark matter particles. In the next section we argue that a widely disseminated solutions to this problem, in terms of boost factors, including those sprouting from Sommerfeld enhancement and similar effects, seems untenable. From this stems our motivation for investigating non-standard cosmological models, allowing for larger DM annihilation cross sections while being less severely constricted by current observations.

\section{PAMELA, ATIC, FERMI and HESS Anomalies}

The PAMELA experiment measured an excess of cosmic ray positrons with no indication of any excess of anti-proton flux. Therefore, if it is indeed the dark matter that is responsible for the positron excess, it seems natural to consider a type of DM particle that annihilates predominantly into $l^{+} l^{-}$channels. The positron flux in the galactic halo is given in terms of the production rate of the positrons from DM annihilation, which is given by

$$
Q(E, \mathbf{r})=\frac{1}{2}\left(\frac{\rho(\mathbf{r})}{m_{\chi}}\right)^{2} \sum_{f}\langle\sigma v\rangle_{f}\left(\frac{d N}{d E}\right)_{f},
$$

where $\langle\sigma v\rangle_{f} \equiv a_{f}$ refers to the averaged annihilation cross section into the final state $f$ and $(d N / d E)_{f}$ is the fragmentation function, representing the number of positrons with energy $E$, produced from the final state $f . \rho(\mathbf{r})$ is a DM halo mass profile. Though there are several types of proposed halo dark matter density profiles, we adopt here the standard NFW profile [15].

Fig. 1 1 shows the total absolute flux, $\Phi_{e^{+}}^{\text {total }}+\Phi_{e^{-}}^{\text {total }}$, generated by the DM annihilation into $e^{+} e^{-}, \mu^{+} \mu^{-}$and $\tau^{+} \tau^{-}$as function of positron energy for $m_{\chi}=1 \mathrm{TeV}$ and $10^{-6} \mathrm{GeV}^{-2}$. 


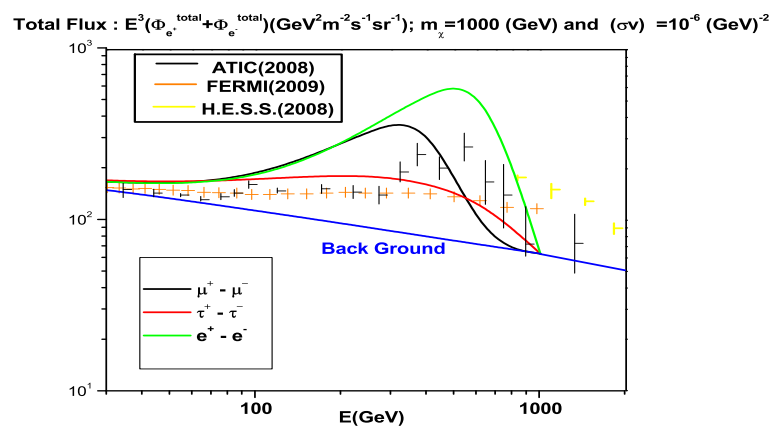

Figure 1: The total absolute flux in units of $\mathrm{GeV}^{2} \mathrm{~m}^{-2} \mathrm{~s}^{-1} \mathrm{sr}^{-1}$, generated by the DM annihilation into $e^{+} e^{-}$(green line), $\mu^{+} \mu^{-}$(black line) and $\tau^{+} \tau^{-}$(red line), as function of positron energy for $m_{\chi}=1 \mathrm{TeV}$, for thermal averaging cross section $10^{-6} \mathrm{GeV}^{-2}$.

In our analysis, the MED diffusion model of Delahaye et. al. 16 and NFW 15 Galactic halo with scale-length $20 \mathrm{kpc}$ are assumed. Also, $\Phi_{e^{+}}^{\text {total }}$ and $\Phi_{e^{-}}^{\text {total }}$ are defined as

$$
\Phi_{e^{+}}^{\text {total }} \sim \Phi_{e^{+}}^{D M}+\Phi_{e^{+}}^{s e c}, \quad \Phi_{e^{-}}^{\text {total }} \sim \Phi_{e^{-}}^{\text {prim }}+\Phi_{e^{-}}^{s e c} .
$$

The flux of positrons $\Phi_{e^{+}}^{D M}$ is given from the number density of positron through several steps(for instance, see ref. [17]).

The fragmentation function for direct process is almost monotonic; $\left(\frac{d N}{d E}\right)_{e e} \sim \delta(E-$ $\left.m_{\chi}\right)$. For $\left(\frac{d N}{d E}\right)_{\mu \mu}$ and $\left(\frac{d N}{d E}\right)_{\tau \tau}$, we referred to the ref. [18].

Calculated in terms of fitting functions matching the fluxes deduced via standard simulations of cosmic ray production and propagation [19] [20], the astrophysical background fluxes of positrons; $\Phi_{e^{+}}^{s e c}, \Phi_{e^{-}}^{\text {prim }}$ and $\Phi_{e^{-}}^{s e c}$, are given by the followings:

$$
\begin{aligned}
\Phi_{e^{-}}^{\text {prim. }}(\epsilon) & =\frac{0.16 \epsilon^{-1.1}}{1+11 \epsilon^{0.9}+3.2 \epsilon^{2.15}}\left(\mathrm{~cm}^{-2} \mathrm{~s}^{-1} \mathrm{sr}^{-1}\right), \\
\Phi_{e^{-}}^{\text {sec. }}(\epsilon) & =\frac{0.70 \epsilon^{-0.7}}{1+110 \epsilon^{1.5}+600 \epsilon^{2.9}+580 \epsilon^{4.2}}\left(\mathrm{~cm}^{-2} \mathrm{~s}^{-1} \mathrm{sr}^{-1}\right), \\
\Phi_{e^{+}}^{\text {sec. }}(\epsilon) & =\frac{4.5 \epsilon^{0.7}}{1+650 \epsilon^{2.3}+1500 \epsilon^{4.2}}\left(\mathrm{~cm}^{-2} \mathrm{~s}^{-1} \mathrm{sr}^{-1}\right),
\end{aligned}
$$

where $\epsilon \equiv E /(1 \mathrm{GeV})$.

We use a DM particle of mass $1 \mathrm{TeV}$, consistent with the maximum allowed by FERMI $\gamma$-ray observations of dark matter dominated virilized gravitational systems [21], [22]. As is immediately apparent, $\Phi_{e^{+}}^{s e c}$ and $\Phi_{e^{-}}^{s e c}$ are quite small at all energies, and can be neglected with respect to $\Phi_{e^{-}}^{\text {prim }}$; the same goes for the computed positron flux for $\langle\sigma v\rangle \sim 10^{-9} \mathrm{GeV}^{-2}$, which remains far below the background. Therefore, in such a situation, and in terms of the plotted quantities, any excess flux would have to be explained in terms of modifications to the astrophysical background (including the possible effects of local pulsars, supernovae remnants etc.). Alternatively, a larger cross section can lift the computed DM flux above the background, so as to explain any apparent excess. It turns out that a $\langle\sigma v\rangle \sim 10^{-6} \mathrm{GeV}^{-2}$ is compatible with PAMELA observations (without invoking any changes in the background 
model). In addition, for positron energy $E>200 \mathrm{GeV}$, the positron flux $\Phi_{e^{+}}^{D M}$ exceeds the electron background, which fares well in explaining the ATIC, HESS and FERMI excess at $300-800 \mathrm{GeV}$.

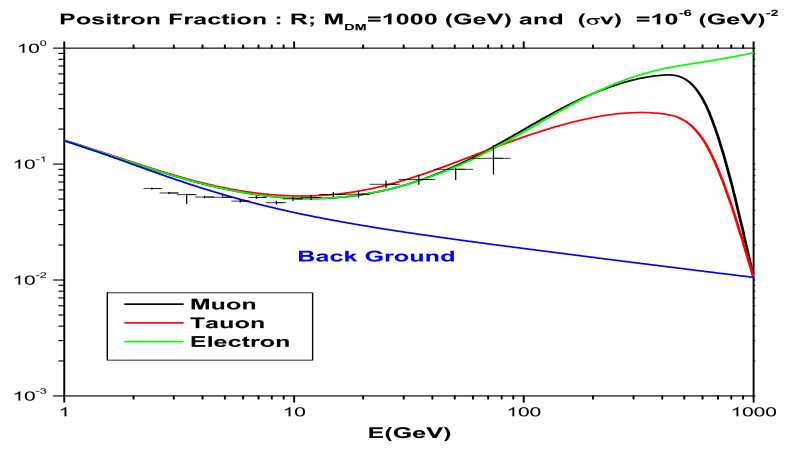

Figure 2: The positron fraction for DM annihilation into $e^{-} e^{+}$(green line), $\mu^{+} \mu^{-}$(black line) and $\tau^{+} \tau^{-}$(red line) for dark matter mass $m_{\chi}=1 \mathrm{TeV}$ and thermal averaging cross section $10^{-6} \mathrm{GeV}^{-2}$.

In Fig. 2, we plot the positron fraction

$$
R=\frac{\Phi_{e^{+}}^{D M}(E)+\Phi_{e^{+}}^{\sec }(E)}{\Phi_{e^{+}}^{D M}(E)+\Phi_{e^{+}}^{\sec }(E)+\Phi_{e^{-}}^{\text {prim }}(E)+\Phi_{e^{-}}^{\text {sec }}(E)},
$$

the quantity that PAMELA actually measured, for DM annihilation into $e^{+} e^{-}, \mu^{+} \mu^{-}$and $\tau^{+} \tau^{-}$, along with the relevant measurements. It is clear, as expected, that DM annihilation with $\langle\sigma v\rangle \sim 10^{-6} \mathrm{GeV}^{-2}$ can easily account for the PAMELA measurements while employing a standard astrophysical background. These measurements explore the existence of positron excess for energies confined between 8 and $80 \mathrm{GeV}$. In the next PAMELA experiment, the search for excess flux will be extended up to energies $\sim 270 \mathrm{GeV}$. We note here that, at such energies, the positron flux due to DM annihilation is of order the electron primary flux and so the expected positron fraction would tend toward

$$
R \simeq \frac{\Phi_{e^{+}}^{D M}}{\left(\Phi_{e^{-}}^{p r i m}+\Phi_{e^{-}}^{D M}\right)+\Phi_{e^{+}}^{D M}} \simeq \mathcal{O}(0.3) .
$$

Such a result would represent a significant signature confirming that DM annihilation is the source of this observed positron excess, making the case for an increased DM flux magnitude (compared to what can be inferred from calculations using standard smooth halo density profiles and cross sections compatible with thermal relic abundance calculations), particularly more pressing.

Already, myriads of remedies, invoking various 'boost factors', have been proposed. Including, for example, in terms of increased DM density inside the galaxy's the subhalo population. Yet very little such substructure is expected in the solar neighborhood; efficient stripping dissolves subhalos in these inner regions, ensuring that most mass in subhalos is concentrated at far larger galactocentric radii. And positrons with energies of order 100 $\mathrm{GeV}$ or above are expected to emanate from DM annihilations within a few hundred pc of 
the solar system. This is quite a generic result, independent of the details of diffusion model adopted for cosmic ray propagation, having its origins in such fairly tractable processes, such as the expected positron energy loss due to inverse Compton scattering off cosmic microwave background [23] and starlight. It is therefore quite unlikely that the required boost in DM annihilation flux can be obtained by invoking the galactic subhalo population - a result confirmed by detailed modelling of its effect [24] [25].

While the existence of other density-borne boosts to the flux cannot, in principle, be completely ruled out (e.g., in addition to the subhalo population, DM caustics, tidal streams, mini-black holes hosting DM spikes etc. have been proposed), a large local enhancement is unlikely to equally boost the positron flux at all energies, and so leave the fits to the flux invariant - again because of the energy dependent manner in which positrons lose energy as they move through the galaxy; indeed, the case for streams and caustics seems to have been falsified, even in principle, i.e. in terms of source function in [26]. The process of fitting the data therefore requires significant fine tuning of masses and positions of objects (as is also the case when invoking the standard subhalo population [27]). Many of these proposals are, in addition, in significant conflict with, or highly constrained by, $\gamma$ ray observations [28] [29].

Another class of proposals involve velocity dependent cross sections originating from Sommerfeld enhancement effects (e.g., [30] [31] [32] [33]). These can dramatically increase annihilation rates at velocities characteristic of galactic halos without affecting the relic abundance. This boost however comes at the price of fine tuning the particle masses [32]; it requires new light scalar or gauge boson, $\phi$, to mediate the annihilation of DM into leptons. The mass of this particle is constrained as:

$$
m_{\phi} \lesssim \alpha m_{\chi} \lesssim \text { few } \mathrm{GeV}
$$

where $\alpha$ refers to the coupling of the $\phi$ interaction squared over $4 \pi$.

Furthermore, proposals for further enhancing the cross section for annihilation by considering the still lower relative particle velocities inside distant subhalos [30], or in the central regions of halo cusps that are shallower than isothermal [34], are untenable, because, again, high energy positrons originating in these regions cannot safely traverse the space separating them from the solar neighborhood.

In addition,Cosmic Microwave background constraints seem to rule out dramatic enhancements due to low dark velocities matter velocities in subhalos, since they suggest the low-velocity annihilation enhancement must have saturated at the last scattering surface (when $v_{\mathrm{DM}} / c \sim 10^{-8}$ ). WMAP results do not, on the other hand, rule out enhanced cross sections consistent with dark matter annihilation from the main halo that explain the cosmic ray excesses. They therefore do not falsify the models described below [35] (though PLANCK results may). Finally, FERMI $\gamma$-ray constraints also seem to rule out enhanced low particle velocity enhanced boosts in subhalos [21] 22].

\section{Relic Abundance and Non-conventional Cosmology}

During the radiation epoch in the early Universe, if the DM is assumed to be in thermal 
equilibrium, at the freeze out temperature $T_{F}$, the standard calculation for the thermal average of the annihilation cross section, for $v \ll c$, yields:

$$
\langle\sigma v\rangle_{F}=a+b v_{F}^{2}
$$

where $v_{F}$ is the velocity of the DM at the freeze out temperature, which is of order $0.1 c$. Note that the above expansion of $\langle\sigma v\rangle$ is consistent only far from s-poles and threshold. In our galactic halo the velocity of the DM particles is much smaller (of order $10^{-3} \times c$ ). Its distribution is also no longer Maxwellian and its dispersion may vary significantly with radius. Nevertheless, due to the suppression of the kinetic energy of the DM respect to its rest mass, the average cross section in the galactic halo has negligible dependence on the velocity distribution function (we have explicitly checked this point by considering the isotropic distribution function [36] associated with the NFW density profile). In this context, $\langle\sigma v\rangle_{\text {halo }}$ can be written as

$$
\langle\sigma v\rangle_{\text {halo }} \simeq a
$$

This implies that the value of $\langle\sigma v\rangle$, involved in the computation of the positron flux in the galactic halo, is the $s$-wave annihilation, which contributes to the DM relic abundance $\Omega h^{2}$.

Given the difficulties outlined above, it seems pertinent to ask whether the constraints imposed on the cross section by standard relic abundance calculations are as universally prohibitive as they seem; and to examine mechanisms whereby they could be circumvented.

The usual assumption is that the DM particles decoupled from the standard model particles when the former became non-relativistic. In that case the DM relic density is given by

$$
\Omega_{\chi} h^{2}=\frac{8.76 \times 10^{-11} \mathrm{GeV}^{-2}}{g_{*}^{1 / 2}\left(T_{F}\right)\left(a / x_{F}+3 b / x_{F}^{2}\right)},
$$

where $T_{F}$ is the freeze out temperature, $x_{F}=m_{\chi} / T_{F} \simeq 20$, and $g_{*}$ the number of relativistic degrees of freedom. It is clear that, for $a \sim 10^{-6} \mathrm{GeV}^{-2}$, one gets $\Omega_{\chi} h^{2} \sim 10^{-4}$, which is inconsistent with the WMAP results [37], as well as a host of observations concerned with the dynamics and large scale distribution of galaxies and clusters.

It is nevertheless important to note that relaxing this assumption can very well give rise to different predictions for the relic density without affecting any of the relevant observations. We will focus here on two such non-standard cosmological scenarios as examples illustrating this point, thus resolving the tension between the relic abundance constraints and the PAMELA and ATIC results. Our first example considers a cosmological scenario where the reheating temperature is associated to the decay of a standard model singlet field $\psi$, in which case the DM relic density considerably increases with respect to the standard radiation-dominated case, by virtue of the effect of direct non-thermal production by the $\psi$ field [38]. The second example is based on the non-conventional brane cosmology [41], which also allows for several orders of magnitude increase in the cross section. The principal point in this first example concerns the numerical relation between the reheating temperature $T_{R H}$ and the the DM freezing temperature $T_{F}$. The former being defined as 
the temperature at which the oscillating field energy ceases to dominate the cosmological evolution, heralding the start of the radiation dominated epoch. In the standard scenario, where $T_{R H} \sim 10^{9} \mathrm{GeV}$, the reheating epoch has no relevance in the final output of the DM relic density. However, for low value of $T_{R H}$, so that $T_{R H}<T_{F}$, it can have important implications on the predictions of the relic abundance of DM as discussed in Ref. 38.

Thus, in this context, it is possible to envision the existence of an epoch in the history of the Universe, preceding the radiation-dominated era, when the energy density was dominated by coherent oscillating fields; the so called reheating era. The decay width of this scalar field $\psi$ can be parameterized via

$$
\Gamma_{\psi}=\frac{1}{2 \pi} \frac{m_{\psi}^{3}}{M_{*}^{2}}
$$

where $M_{*}$ defines a high scale, acting as an effective suppression scale.

The coupled Boltzmann equation, for the DM, scalar field $\psi$ and radiation, is then solved. The detailed equations used in this computation can be found in Ref. [38]. The resulting relic density can then be estimated to be [39] 440]

$$
\Omega_{\chi} h^{2} \sim\left(\frac{M_{*}}{1.5 \times 10^{20} \mathrm{GeV}}\right)\left(\frac{1 \mathrm{GeV}^{-2}}{\langle\sigma v\rangle}\right)\left(\frac{100 \mathrm{GeV}}{m_{\psi}}\right)^{3 / 2}\left(\frac{10.75}{g_{*}}\right)^{1 / 4}\left(\frac{m_{\chi}}{100 \mathrm{GeV}}\right) .
$$

From this equation, it can be seen that it possible to obtain $\Omega_{\chi} h^{2} \sim \mathcal{O}(0.1)$ with an annihilation cross section of order $10^{-6} \mathrm{GeV}^{-2}$, as required by PAMELA and ATIC data. For example, for :

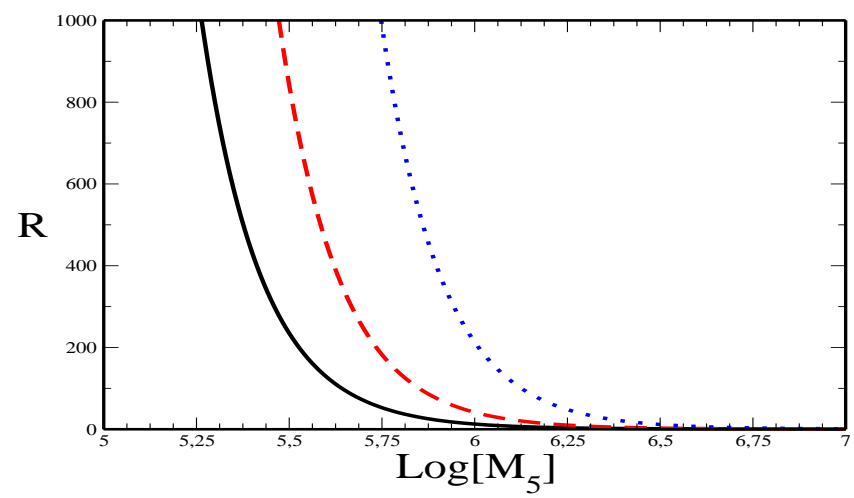

Figure 3: The enhancement/suppression factor $R=\left(\Omega_{\chi} h^{2}\right)_{b} /\left(\Omega_{\chi} h^{2}\right)_{s}$ as a function of the five dimensional scale $M_{5}(\mathrm{GeV})$ for $m_{\chi}=100$ (solid curve), 200 (dashed curve) and $500 \mathrm{GeV}$ (dotted curve).

We now turn to the second possible scenario invoking non-standard cosmology as a mechanism for boosting the DM annihilation cross section. As mentioned above, it is based on the brain world cosmology, which is embedded in five dimensional warped space time. In this case, the derived Friedman equation is given by

$$
H^{2}=\frac{8 \pi G_{(4)}}{3} \rho\left(1+\frac{\rho}{2 \sigma}\right)-\frac{k}{a^{2}}+\frac{\mathcal{C}}{a^{4}},
$$


where $H=\dot{a} / a$ is the Hubble parameter and $a(t)$ is the scale factor, $\rho$ is the energy density of ordinary matter on the brane, while $\sigma$ is the brane tension; $G_{(4)}$ refers to the $4 D$ Newton coupling constant, $k$ stands for the curvature of our three spatial dimensional and $\mathcal{C}$ is a constant of integration known as dark-radiation. This equation implies that $H \propto \rho$ rather than $\sqrt{\rho}$ as in the standard cosmology. Thus, the evolution of the scale factor will be different from the usual one. This modification affects any relic abundance of DM that may be reminiscent of the radiation dominated phase of the early Universe [41] [42] [43]. For, in this model, the Universe undergoes a nonstandard brane cosmology at early times till it reaches a temperature, known as transition temperature $T_{t}$, when it sustains the standard cosmology. This transition temperature is defined as

$$
\rho\left(T_{t}\right)=2 \sigma \quad \Rightarrow \quad T_{t}=0.51 \times 10^{-9} M_{5}^{\frac{3}{2}} \mathrm{GeV},
$$

and the transition should take place above the nucleosynthesis era (i.e., $T_{t}>1 \mathrm{MeV}$ ). Here $M_{5}$ is the five dimensional Plank mass. If the freeze out temperature of the DM $\left(T_{F}\right)$ is higher than the transition temperature, i.e., $T_{F} \geq T_{t}$ and $M_{5} \leq 10^{5} \mathrm{GeV}$, one finds that the ratio between the relic density in brane and standard cosmology is given by [41]

$$
R=\left(\Omega_{\chi} h^{2}\right)_{b} /\left(\Omega_{\chi} h^{2}\right)_{s} \simeq \mathcal{O}\left(10^{2}-10^{3}\right) .
$$

In Fig. 3, we present the prediction for the factor $R$ as a function of the scale of the five dimensions, $M_{5}$, for different values of $m_{\chi}$, namely we consider $m_{\chi}=100,200$ and $500 \mathrm{GeV}$. As can be seen from this figure, for $M_{5}<10^{6}$ the brane cosmology effect is quite large and the factor $R$ becomes much larger than one. In this case the resulting relic density $\left(\Omega_{\chi} h^{2}\right)_{b}$ may exceed the WMAP results $\Omega_{\chi} h^{2} \simeq 0.1$. Moreover for $M_{5} \gtrsim 5 \times 10^{6}$, the ratio $R$ becomes less than one and a small suppression for $\left(\Omega_{\chi} h^{2}\right)_{s}$ can be obtained.

An analogous analysis, in the case of standard cosmology, would give $\left(\Omega h^{2}\right)_{s} \simeq 10^{-3}$, under the fixed parameters $m_{\chi}=1 \mathrm{TeV},\langle\sigma v\rangle=10^{-6}$. Hence, for this calculation to be consistent with $\left(\Omega h^{2}\right)_{b} \simeq 0.1, R$ must reach at around $10^{2}$. Hence $M_{5}$, in our case, has to be more than $10^{6}$ from the Fig. 3 .

This brane enhancement or suppression for the dark matter relic density could be favored or disfavored based on the value of the relic abundance in the standard scenario. If $\left(\Omega_{\chi} h^{2}\right)_{s}$ is already larger than the observational limit, as in the case of bino-like particle, then a suppression effect would be favored and hence $M_{5}$ is constrained to be larger than $5 \times 10^{6} \mathrm{GeV}$. However, for wino- or Higgsino-like particle where the standard computation usually leads to very small relic density, the enhancement effect will be favored and the constraint on $M_{5}$ can be relaxed a bit [44]. In general, it is remarkable that in this scenario the dark matter relic density imposes a stringent constraint on the fundamental scale $M_{5}$. This brane enhancement implies that stable particles with annihilation cross section $\sim 10^{-6} \mathrm{GeV}^{-2}$, which seems essential for accommodating the PAMELA and ATIC results, remain viable $\mathrm{DM}$ candidates. 


\section{Extra Constraints on the Annihilation Cross Section and the Viability of our Model}

For our model to be viable, it has to be consistent with other constraints. Some of these were briefly mentioned earlier in our discussion; we summarize the situation as regards to these here.

DM annihilation at redshift $z \sim 1000$ has an effect on the degrees of ionization of the primordial plasma, by virtue of the energy it pumps into it through the high energy particles it produces. By affecting its degree of ionization, it broadens the last scattering surface, which in turn has an effect on temperature correlations and polarization of the cosmic microwave background. Slatyer et. al. [35] have presented a detailed study of the phenomenon. Their results tend to disfavor the large boosts implied by Sommerfeld enhancements in dark matter substructure, but not as much the standard enhancement at normal local dark halo velocities. WMAP results therefore do not rule out the cross section boosts required for fitting the PAMELA and ATIC results in the context of nonstandard cosmological scenarios, and are even less severe in constraining the FERMI data. Though further constraints from PLANCK would provide further stringent tests that may in principle falsify the scenarios presented here.

Since dark matter annihilation produces secondary gamma rays, through internal bremsstrahlung and inverse Compton scattering, there are also severe constraints on DM annihilation cross sections from the observed gamma ray fluxes; in particular from recent observations by FERMI . The most stringent appear to be those concerning the gamma rays emanating dark matter dominated systems such as dwarf galaxies and galaxy clusters.Recently published results from eleven months of FERMI observations [21], 22], seem to disfavor cross sections consistent with the observed cosmic ray excesses if the particles producing these fluxes exceed the $1 \mathrm{TeV}$ limit, particularly if the annihilation proceeds through the muon channel. Thus all the models presented here are (albeit in one case marginally) consistent with these constraints.

Finally, the current experimental limits from CDMS [45] and XENON10 [46] imply that the DM-nucleus scattering cross section is less than $10^{-7} \mathrm{GeV}^{-2}$. By crossing symmetry in Quantum Field Theory (QFT), the annihilation cross section and the scattering cross section are related; which may lead one to suspect the presence of a contradiction between the direct detection results and the attempt to explain the PAMELA and ATIC data via enhanced annihilation cross sections. But this apparent conflict can be resolved by assuming that the DM particle has suppressed coupling with quarks, while retaining reasonably large coupling with leptons; i.e., that the DM has a leptonic nature. As mentioned above, this type of DM is also favored for explaining the absence of flux excess in the observed anti-proton flux in PAMELA, ATIC and FERMI data. It is clear that in this case one can naturally have the annihilation cross section into $l^{+} l^{-}$of order $10^{-6} \mathrm{GeV}^{-2}$, whereas the scattering cross section of DM and nucleus $\sigma_{\mathrm{DM}-\mathrm{N}}$ is quite suppressed.

It is also important to note that an $s$-wave dominated annihilation cross section of order $10^{-6} \mathrm{GeV}^{-2}$ bears interesting corollaries concerning the nature of prospective DM candidates, and by implication on particle physics theory. It is well known, for exam- 
ple, that if the DM is composed of Majorana particles, then its annihilation cross section into leptons is proportional to $m_{l}^{2} / m_{\chi}^{4}$; which, for $m_{\chi} \simeq 100 \mathrm{GeV}$, is typically less than $10^{-8} \mathrm{GeV}^{-2}$. However, for Dirac or scalar boson type DM the annihilation cross section is no longer proportional to the lepton mass squared and it may thus be enhanced significantly. In this context, the famous DM candidate of lightest neutralino in minimal supersymmetric standard model, which is a Majorana particle, is not favored for explaining the PAMELA/ATIC results. On the other hand, the right-handed sneutrino, which is considered an interesting scalar candidate for DM in supersymmetric theories with righthanded neutrino [47, can account for the large annihilation cross sections required for explaining the PAMELA/ATIC measurements.

\section{Conclusions}

In this paper we have proposed solutions reconciling the interpretation of the observed cosmic ray excesses with dark matter annihilation models by invoking non-standard cosmologies. We have argued that these models have several advantages over those invoking density or velocity borne boost factors, especially those invoking large enhancements due to dark matter overdensities and halo substructures. At present, our models are consistent with current observations. Future data, especially those from the PLANCK concerning the enhanced effect of DM annihilation on the Cosmic Microwave Background, will determine whether our models remain viable.

\section{Acknowledgments}

This work was partially supported by the ICTP Project ID 30, Science and Technology Development Fund (STDF) Project ID 437, and the Egyptian Academy for Scientific Research and Technology.

\section{References}

[1] R. Bernabei et al. [DAMA Collaboration], Eur. Phys. J. C 56, 333 (2008).

[2] Z. Ahmed et al. [The CDMS-II Collaboration], arXiv:0912.3592 [astro-ph.CO].

[3] O. Adriani et al. [PAMELA Collaboration],

[4] S. W. Barwick et al. [HEAT Collaboration], Astrophys. J. 482 (1997) L191; J. J. Beatty et al., Phys. Rev. Lett. 93 (2004) 241102.

[5] M. Aguilar et al. [AMS-01 Collaboration], Phys. Lett. B 646, 145 (2007).

[6] J. Chang et al., Nature 456 (2008) 362.

[7] T. Delahaye, F. Donato, N. Fornengo, J. Lavalle, R. Lineros, P. Salati and R. Taillet, Astron. Astrophys. 501, 821 (2009) [arXiv:0809.5268 [astro-ph]].

[8] N. J. Shaviv, E. Nakar and T. Piran, Phys. Rev. Lett. 103, 111302 (2009) [arXiv:0902.0376 [astro-ph.HE]].

[9] S. Profumo, arXiv:0812.4457 [astro-ph]. 
[10] M. Cirelli, M. Kadastik, M. Raidal and A. Strumia, Nucl. Phys. B 813, 1 (2009) [arXiv:0809.2409 [hep-ph]].

[11] D. Hooper, arXiv:0901.4090 [hep-ph].

[12] Fermi/Lat Collaboration, Phys.Rev.Lett .102, 181101 (2009).

[13] F. Aharonian et al. [H.E.S.S. Collaboration], Phys. Rev. Lett. 101, 261104 (2008).

[14] H. E. S. Aharonian, Astron. Astrophys. 508, 561 (2009) [arXiv:0905.0105 [astro-ph.HE]].

[15] Julio F. Navarro, Carlos S. Frenk, Simon D.M. White, Astrophys.J.490:493-508,1997.

[16] T. Delahaye, R. Lineros, F. Donato, N. Fornengo, P. Salati, Phys.Rev.D. 77:063527, 2008.

[17] J. Hisano, S. Matsumoto, O. Saito and M. Senami, Phys. Rev. D 73, 055004 (2006) [arXiv:hep-ph/0511118].

[18] C. Pallis, Nucl. Phys. B 831, 217 (2010) [arXiv:0909.3026 [hep-ph]].

[19] E. A. Baltz and J. Edsjo, Phys. Rev. D 59, 023511 (1998) [arXiv:astro-ph/9808243].

[20] T. Delahaye, F. Donato, N. Fornengo, J. Lavalle, R. Lineros, P. Salati and R. Taillet, Astron. Astrophys. 501, 821 (2009) [arXiv:0809.5268 [astro-ph]].

[21] FERMI-LAT Collaboration, arXiv1002.2239 [astro-ph]

[22] A. A. Abdo, et al. [Fermi-LAT Collaboration], Astrophys. J., accepted (2009) [arXiv:1001.4531], A. A. Abdo et al. [The Fermi LAT Collaboration], Phys. Rev. Lett. 102, 181101 (2009) [arXiv:0905.0025 [astro-ph.HE]].

[23] T. R. Slatyer, N. Padmanabhan and D. P. Finkbeiner, Phys. Rev. D 80, 043526 (2009) [arXiv:0906.1197 [astro-ph.CO]].

[24] J. Lavalle, Q. Yuan, D. Maurin, Y.- J. Bi, Astron. \& Astrophys. 479, 427.

[25] J. Lavalle, E. Nezri, F.-S. Ling, E. Athanassoula, R. Teyssier, Phys. Rev. D. 78103526.

[26] M. Vogelsberger, S. D. M. White, arXiv1002.3162 [astro-ph]

[27] D. Hooper, A. Stebbins and K. M. Zurek, Phys. Rev. D 79, 103513 (2009) [arXiv:0812.3202 [hep-ph]].

[28] T. Bringmann, J. Lavalle and P. Salati, Phys. Rev. Lett. 103, 161301 (2009) [arXiv:0902.3665 [astro-ph.CO]].

[29] G. Bertone, M. Cirelli, A. Strumia and M. Taoso, JCAP 0903, 009 (2009) [arXiv:0811.3744 [astro-ph]].

[30] M. Lattanzi and J. I. Silk, Phys. Rev. D 79, 083523 (2009) [arXiv:0812.0360 [astro-ph]].

[31] N. Arkani-Hamed, D. P. Finkbeiner, T. R. Slatyer, N. Weiner, Phys. Rev. D7, 015014 ( 2009).

[32] A. Decourchelle, Astron. Nachr. AN 329, 178 (2008).

[33] J. Hisano, S. Matsumoto, M. M. Nojiri and O. Saito, Phys. Rev. D 71, 063528 (2005) [arXiv:hep-ph/0412403].

[34] B. Robertson and A. Zentner, Phys. Rev. D 79, 083525 (2009) [arXiv:0902.0362 [astro-ph.CO]].

[35] T. R. Slatyer, N. Padmanabhan, D. P. Finkbeiner, Phys. Rev. D80, 043526 (2009). 
[36] Lawrence M. Widrow, Astrophysical Journal, arXiv:astro-ph/0003302

[37] G. Hinshaw et al. [WMAP Collaboration], Astrophys. J. Suppl. 180, 225 (2009).

[38] S. Khalil, C. Munoz and E. Torrente-Lujan, New J. Phys. 4, 27 (2002).

[39] T. Moroi and L. Randall, Nucl. Phys. B 570, 455 (2000) [arXiv:hep-ph/9906527].

[40] S. Mizuta and M. Yamaguchi, Phys. Lett. B 298, 120 (1993) [arXiv:hep-ph/9208251].

[41] E. Abou El Dahab and S. Khalil, JHEP 0609, 042 (2006).

[42] L. Randall and R. Sundrum, Phys. Rev. Lett. 83, 4690 (1999) [arXiv:hep-th/9906064].

[43] N. Okada and O. Seto, Phys. Rev. D 70, 083531 (2004) [arXiv:hep-ph/0407092].

[44] T. Nihei, N. Okada and O. Seto, Phys. Rev. D 73, 063518 (2006) [arXiv:hep-ph/0509231].

[45] CDMS Collaboration, Phys. Rev. Lett. 102:011301,2009.

[46] XENON Collaboration, Phys. Rev. Lett. 100:021303,2008.

[47] Chiara Arina, Nicolao Fornengo, JHEP 0711:029,2007. 